\title{
Microbial Contamination of Extended Use Ophthalmic Drops in Ophthalmology Clinic
}

\author{
Shee Wen Chua $\mathbb{D}^{1,2}$ \\ Mushawiahti Mustapha' \\ Kon Ken Wong ${ }^{3}$ \\ Malisa $\mathrm{Ami}^{4}$ \\ Aida Zairani Mohd Zahidin ${ }^{5}$ \\ Rona Asnida Nasaruddin ' \\ 'Department of Ophthalmology, Faculty \\ of Medicine, Universiti Kebangsaan \\ Malaysia, Kuala Lumpur, Malaysia; \\ ${ }^{2}$ Ophthalmology Department, Faculty of \\ Medicine and Health Science, Universiti \\ Malaysia Sabah, Kota Kinabalu, Sabah, \\ Malaysia; ${ }^{3}$ Department of Medical \\ Microbiology and Immunology, Faculty of \\ Medicine, Universiti Kebangsaan Malaysia, \\ Kuala Lumpur, Malaysia; ${ }^{4}$ Sunway \\ Specialist Center Damansara, Petaling \\ Jaya, Selangor, Malaysia; ${ }^{5}$ Gleneagles \\ Hospital, Kuala Lumpur, Kuala Lumpur, \\ Malaysia
}

Purpose: The objectives of this study were to determine the prevalence of microbial contamination of multi-user preserved ophthalmic drops (POD) in Ophthalmology Outpatient Clinic (OOC), to compare the rate of contamination between the dropper tip and the residual contents in the bottle, and to identify the contaminating organisms.

Methods: This was an observational cross-sectional study using a convenience sampling method conducted in the OOC of Universiti Kebangsaan Malaysia Medical Center, Malaysia. The samples of POD bottles were divided into groups obtained after 14 days (T14) and after 30 days (T30) of use. The contamination rate at the dropper tip and in the residual contents was determined and the contaminating organisms were identified.

Results: A total of 140 of 149 extended-use POD bottles were included. The prevalence of contamination was $30 \%$. There was a statistically significant difference in the rate of contamination between samples $\mathrm{T} 14$ and $\mathrm{T} 30$ ( $19 \%$ and $11 \%$, respectively; $p=0.046)$. Proparacaine and tropicamide showed higher contamination rates in the T14 samples ( $p=0.027$ and $p=0.497$, respectively) than in the T30 samples. The site of contamination was higher at the dropper tip than in the residual contents $(p>0.05)$. Coagulase-negative Staphylococcus species were the most frequently identified contaminants (89\%).

Conclusion: The dropper tip was more contaminated than the residual contents, and coagulase-negative Staphylococcus species, which are common commensal flora of the ocular conjunctiva and skin, were the most frequently identified organisms.

Keywords: contamination rate, extended period, preserved ophthalmic drops, multi-user

\section{Introduction}

In ophthalmology outpatient clinics (OOC), a single bottle of preserved ophthalmic drops (POD) is often used on multiple patients ${ }^{1,2}$ over an extended period of time in order to decrease the cost to the hospital. ${ }^{3-7}$ In the OOC of Universiti Kebangsaan Malaysia Medical Center (UKMMC), Malaysia, ophthalmic drops are discarded once or twice a month regardless of how much content remains. Some of the frequently used PODs are Alcaine $0.5 \%$ (proparacaine hydrochloride) $(15 \mathrm{~mL}$ dispenser), Mydriacyl 1\% (tropicamide) (15 mL dispenser), and Mydfrin 2.5\% (phenylephrine hydrochloride) ( $8 \mathrm{~mL}$ dispenser). All three are manufactured by Alcon $^{\circledR}$ [Novartis Corporation (Malaysia) Sdn. Bhd., Petaling Jaya, Malaysia] and have a similar preservative, benzalkonium chloride (B.A.K) $(0.01 \%)$, to prevent contamination via inhibiting the growth of microorganisms by interfering with their metabolism. ${ }^{8,9}$ However, few studies have shown that the addition of B.A.K or thimerosal to ophthalmic drops is not sufficient to ensure its sterility. ${ }^{4,10}$ In particular, the use of these drops in multiple patients and over an extended period of
Correspondence: Mushawiahti Mustapha Department of Ophthalmology, Faculty of Medicine, Universiti Kebangsaan Malaysia Medical Center, Kuala Lumpur, 56000, Malaysia

Tel +6039l45598I

Email drmusha@gmail.com 
time carries a potential risk of avoidable ocular infections and cross contamination. ${ }^{9,11,12}$

In addition, there is a risk of contamination at any point during the handling of ophthalmic drops, including during drop instillation. ${ }^{4,13,14}$ A multi-use eyedrops policy has been established in Murray, Utah, to provide instructions for the safe handling and administration of eyedrops that could reduce the risk of contamination. ${ }^{1}$

The reported contamination rate in previous studies varied widely from 2.2 to $70 \% .^{3-6,9,13}$ In the OOC of UKMMC, Malaysia, ophthalmic drops are discarded once or twice a month regardless of how much content remains. However, the contamination rate in the OOC of UKMMC is currently unknown. There is also no published study on the contamination rate associated with the use of POD in multiple patients in Malaysia. The literature reviews were mainly from temperate countries, but Malaysia is a tropical country. ${ }^{1,3,12}$ As the contamination rate may vary depending on the environmental conditions, it is important to study and understand the contamination rate in tropical countries as well.

The objectives of this study were to determine the prevalence of microbial contamination of multi-user POD in OOCs in our institution, to compare the contamination rate between the dropper tip and residual contents in the bottle and to identify the contaminating organisms.

\section{Methods}

This was an observational cross-sectional study conducted on topical ophthalmic drops used in the UKMMC OOC. The PODs tested were Alcaine 0.5\% (proparacaine hydrochloride) (15 mL dispenser) and Mydriacyl 1\% (tropicamide) (15 $\mathrm{mL}$ dispenser). They are both manufactured by Alcon ${ }^{\circledR}$ and have a similar preservative composition of $0.01 \%$ of B.A.K.

The sample size was calculated using the convenience sampling method, based on the assumption of a contamination rate of $10 \%$ as determined in a similar study by Livingstone et al United Kingdom, which found a contamination rate of $9.1 \%$ at 14 days. ${ }^{5}$ The number of samples required to adequately compare the contamination rate of the dropper tips and the residual contents was determined using Cohen's effect size with a power of $80 \%$ at a two-tailed $\alpha$ value of 0.5 and a standard deviation of $50 \%$. According to the calculation, at least 64 samples were required for each arm.

The inclusion criteria for this study were newly opened POD bottles $(15 \mathrm{~mL}$ dispensers of Alcaine $0.5 \%$ and
Mydriacyl 1\%) used in OOC. Each bottle was randomly assigned a number $(1,2,3, \ldots$ to 149$)$, and this number was used to label the bottle and its cap. The dropper tip of each newly opened bottle was swabbed with a dry swab under aseptic conditions. This sample was considered as the control sample (T0). It was placed in a nutrient broth, which was used as a transport medium, and cultured on a nutrient agar plate in the microbiology laboratory. Control samples cultured on the nutrient agar plate were monitored for up to 8 days, and bottles found to be contaminated at the beginning of the study were excluded from the study and discarded. Non-contaminated bottles continued to be used on patients for an extended period of time. Labeled PODs were collected prior to discarding from the OOC. Based on the opening and discard dates, the collected PODs were conveniently categorized into 14day used (T14 samples) or 30-day used (T30 samples). Exclusion criteria included a broken seal on new bottles, contamination of the control sample (T0), a missing cap or tip on the day of collection, and bottles used in cases of infection. A total of 72 samples of T14 and 68 samples of T30 were collected.

The same investigator took the sample swab from the new bottle (T0) and on the days of collection (T14 or T30). The dry swab was placed in a nutrient broth used as a transport medium before being sent to the microbiology laboratory for culture at all two time points. In addition, at T14 or T30, the ophthalmic drop bottles were sent to the microbiology laboratory. A total of 140 samples of nutrient broth (Thermo Fisher Scientific, USA) containing the dry swab and the residual contents of the 140 bottles were inoculated onto blood agar, chocolate agar, MacConkey agar, and Sabouraud dextrose agar plates (Thermo Fisher Scientific, USA). All culture plates were incubated in an incubator (Memmert, Germany) with $5 \% \mathrm{CO}_{2}$ at $37^{\circ} \mathrm{C}$. Cultures were evaluated daily for growth by the same microbiologist until day 8 inoculation. When positive growth was observed on the culture agar plates, Gram staining was performed and observed under the microscope to determine Gram-negative and Gram-positive bacteria. Gram-positive cocci bacteria were identified based on the result of catalase and coagulase reactions. Gram-negative bacteria were further tested with a series of biochemical assays using triple sugar iron, urea, citrate and motility. Analysis of the observed biochemical reactions was used to identify the causative organism. ${ }^{15}$ All samples of nutrient broth and bottles were subsequently discarded. 
IBM Statistical Package for the Social Sciences (SPSS) software version 26 was used. Descriptive statistics were used to analyze the data. Pearson's Chi-square test was used to assess differences in contamination rates at T14 and T30, and the general linear regression test was used to assess contamination risk. $P$-values of $<0.05$ were considered to indicate statistical significance.

\section{Results}

Of the original 149 ophthalmic drop bottles, 140 were finally included in the analysis: Four bottles in the control group (T0) were excluded because they were contaminated, and five labeled bottles were missing on the day of collection.

As shown in Table 1, there were no statistically significant intergroup differences between the T14 and T30 samples in terms of the type of ophthalmic drops used (Alcaine vs Mydriacyl).

The overall prevalence of contamination was 30\% (42/ 140), as shown in Table 2. Cumulatively, there was a significant difference in the rate of contamination between the T14 (19\%) and T30 (11\%) samples $(p=0.046)$. Proparacaine was associated with a significantly higher contamination rate in the T14 samples than in the T30 samples $(20 \%$ vs $7 \%$; $p=0.027)$, but the tropicamide eyedrop showed no significant difference

Table I Distribution of Multi-User PODs in 2 Extended Periods of Usage

\begin{tabular}{|l|l|l|l|}
\hline PODs & TI4 (n,\%) & T30 (n,\%) & p-value \\
\hline Type of PODs & & & 0.862 \\
Proparacaine & $36(25.7)$ & $33(23.6)$ & \\
Tropicamide & $36(25.7)$ & $35(25.0)$ & \\
Total $(n=140)$ & $72(51.4)$ & $68(48.6)$ & 0.114 \\
\hline
\end{tabular}

Notes: T14, Ophthalmic drops used for 14 days; T30, Ophthalmic drops used for 30 days.

Table 2 PODs Contamination in 2 Extended Periods of Usage

\begin{tabular}{|c|c|c|c|}
\hline PODs & TI4 (n,\%) & T30 (n,\%) & $p$-value \\
\hline Contamination & $27(19.3)$ & $15(10.7)$ & $0.046 *$ \\
\hline Type of PODs & & & 0.248 \\
\hline Proparacaine $^{+}$ & $14(20.3)$ & $5(7.2)$ & $0.027^{*}$ \\
\hline Tropicamide $^{+}$ & $13(18.3)$ & $10(14.1)$ & 0.497 \\
\hline Sites of contamination & & & 0.901 \\
\hline Dropper tip ${ }^{+}$ & $13(31.0)$ & $8(19.0)$ & 0.747 \\
\hline Residual contents $^{+}$ & $9(21.4)$ & $5(11.9)$ & 1.000 \\
\hline Both sites $^{+}$ & $5(11.9)$ & $2(4.8)$ & 0.666 \\
\hline
\end{tabular}

Notes: ${ }^{+}$Contamination; $*$-value statistically significant $<0.05$ using Pearson's Chisquare test. in its contamination rate between the T14 and T30 samples $(p=0.497)$.

The dropper tip was associated with a much higher contamination rate than the residual bottle contents $(50 \%$ vs $33 \% ; p>0.05) .17 \%$ of samples had contamination at both sites. There was no significant difference between sites of contamination in any POD.

Table 3 reveals that the contaminants identified were mainly coagulase-negative Staphylococcus sp. (CONS) $(89 \%)$, followed by gram-negative rod species $(7 \%)$. Other contaminants were Micrococcus sp. and Acinetobacter sp. (4\%).

Table 4 shows that there was a $3 \%$ (Cox \& Snell $\left.\mathrm{R}^{2}=0.031, p=0.041\right)$ predictor of risk of POD contamination using a simple logistic regression test. The result showed that a longer period of POD use was a negative and marginally significant $(B=-0.757, \quad$ S.E. $=0.381$, $p=0.047)$ predictor of POD contamination. However, no significance was found for the types of PODs.

\section{Discussion}

The prevalence of contamination rate in the UKMMC OOC was $30 \%$. The contamination rate reported in other studies varies considerably (2.2\% to $70 \%){ }^{3-6,9,13}$ A study in the United Kingdom with a total of 636 samples of eyedrops from bottles used in multiple patients and over a long period of time found that there was little to no association between contamination rate and the duration of bottle use. ${ }^{5}$ They reported that the contamination rate was $6 \%$ at 7 -day use and $9 \%$ at 14 -day use $(p>0.1){ }^{5}$ Based on their results, they concluded that a single bottle of eyedrops can be used for up to 14 days without posing a risk to patient health. ${ }^{5}$ Our study found a higher contamination rate at $\mathrm{T} 14$. The difference between the studies could be due to other factors associated with microbial contamination such as handling technique, hygiene practices, instillation angle, and bottle geometry. ${ }^{6,711}$ In

Table 3 Identified Contaminants from the Contaminated PODs

\begin{tabular}{|l|c|c|c|}
\hline $\begin{array}{l}\text { Identified } \\
\text { Contaminants }\end{array}$ & $\begin{array}{c}\text { TI4 } \\
\text { (n) }\end{array}$ & $\begin{array}{c}\text { T30 } \\
\text { (n) }\end{array}$ & $\begin{array}{c}\text { Total, } \mathbf{n = 4 2} \\
\text { (\%) }\end{array}$ \\
\hline CONS & 25 & 12 & $37(89 \%)$ \\
\hline Other organisms & & & \\
$\quad$ Micrococcus sp. & $\mathrm{I}$ & 0 & $\mathrm{I}(2 \%)$ \\
Gram-negative rod & $\mathrm{I}$ & 2 & $3(7 \%)$ \\
Acinetobacter sp. & 0 & $\mathrm{I}$ & $\mathrm{I}(2 \%)$ \\
\hline
\end{tabular}

Note: $p$-value CONS with others organisms using Pearson's Chi-square test: 0.227 . Abbreviations: CONS, coagulase-negative Staphylococcus species; sp., species. 
Table 4 Association Between Type of Eyedrops and Extended Period Usage of Eyedrops with Contamination Rate

\begin{tabular}{|l|l|l|l|l|}
\hline Contamination & B (S.E.) & Wald $^{\mathbf{a}}$ (df) & OR (95\% Cl) & p-value \\
\hline Type of PODs & $0.250(0.376)$ & $0.443(\mathrm{I})$ & $0.393(0.615,2.68 \mathrm{I})$ & 0.506 \\
Extended period of usage & $-0.757(0.38 \mathrm{I})$ & $3.946(\mathrm{I})$ & $3.971(0.222,0.990)$ & $0.047^{*}$ \\
Constant & & $4.193(\mathrm{I})$ & 4.402 & Ref. \\
\hline
\end{tabular}

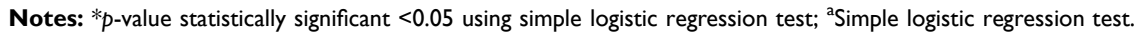

Abbreviations: B, coefficient for the constant; S.E., standard error; df, degree of freedom for each variables; OR, odd ratio; Ref., reference.

addition, the previous study was conducted in a temperate country while our study was conducted in a tropical country.

We studied two types of eyedrops - Alcaine (proparacaine hydrochloride) and Mydriacyl (tropicamide) containing similar percentage of preservative B.A.K. and manufactured by the same company. Proparacaine is commonly used as a local anesthetic and in examinations such as measurement of intraocular pressure and fluorescein staining, whereas tropicamide is less commonly used in the clinic for pupillary dilation. ${ }^{4,9,13}$ In addition, proparacaine is usually instilled by the examining clinician under a slit lamp, whereas tropicamide is usually instilled by a clinic assistant or nurse while the patient is sitting in the waiting area. Therefore, the contamination rate between these two eyedrops may vary depending on the frequency of use and handling technique. In our study, similar results were found as in studies from Kenya and Ethiopia, ${ }^{4,9}$ where contamination rates were higher for bottles of local anesthetic than mydriatics, which was explained by the frequent use of anesthetic drops. Literature reviews reported that tetracaine or proparacaine ( $10 \%$ to $20 \%)$ had a higher contamination rate than mydriatics $(5 \%$ to $7 \%)$. ${ }^{9,13}$

Our study found that the dropper tips had a higher contamination rate than the residual bottle contents, although the difference was not significant. This is in agreement with the findings of other published reports, ${ }^{6,8,13}$ except for one published study which reported opposite results. ${ }^{9}$ It is expected that the dropper tip is more contaminated as it is the most exposed part and comes in direct contact with the patient's eye. Although B. A.K. has antimicrobial activity, the contact time at the dropper tip was not sufficient for the preservative to exert its antimicrobial activity. ${ }^{4,5}$ In addition, a few studies have shown that the addition of B.A.K. or thimerosal to ophthalmic drops is not sufficient to ensure their sterility. ${ }^{4,10}$

Poor technique in the administration of ophthalmic drops is considered a potential contamination risk, even when instilled by healthcare professionals. ${ }^{4,12,16}$ The risk of contamination is increased by physical contact with the dropper tip while opening the bottle, contact of the tip with ocular tissue, and exposure to environmental contaminants if the bottle is left uncapped. ${ }^{4}$ Because proparacaine is an anesthetic, it is more commonly used when examining patients (measuring intraocular pressure or performing the Seidel's test) or before a minor procedure such as removing a suture. ${ }^{4,9,13}$ The examining clinician may instill proparacaine under a slit lamp, and there is a high likelihood that the dropper tip will come into physical contact with the patient's ocular tissue or adnexa. There is also the possibility of the dropper tip accidentally coming into contact with the slit lamp surface during drop instillation. All of this may explain the higher contamination rate associated with proparacaine.

Our study had shown that the commonly identified contaminants were resident flora found in the conjunctiva, eyelid, and skin consisting mainly of Gram-positive bacteria, including coagulase-negative Staphylococcus sp. and Micrococcus sp. and a smaller percentage of Gramnegative rod bacteria. This suggests that the contaminated ophthalmic drops may have come into contact with the ocular surface of patients or the fingers/hands of health care professionals, as reported in previous studies. ${ }^{4,12,16}$

In 2012, a multi-use eyedrops policy was established in Murray, Utah, which included instructions for safe handling and administration of eyedrops to reduce the risk of contamination. ${ }^{1}$ To further reduce the rate of contamination of PODs at our center, the guidelines can be adopted as part of the hygiene protocol for instillation of ophthalmic drops: wash hands thoroughly before administering eyedrops, tilt the patient's head slightly back, pull the lower eyelid away from the eye to form a pocket, instruct the patient to look up, place the dropper directly over the eye, and avoid contact between the dropper tip and any part of the eyelid, eyelashes, or the eye itself. ${ }^{1}$ Regular, periodic review of the drop instillation protocol may be beneficial in reducing the rate of POD contamination. 
Other factors have also been shown to contribute to a lower rate of POD contamination. It has been reported that increasing the instillation angle of PODs to 90 degrees instead of 45 degrees decreases the contamination rate from $83 \%$ to $22 \%{ }^{11}$ In addition, PODs with rounded nozzle tips have been reported to fail to prevent the flow of solution to the side of the bottle and have a higher contamination rate than smaller nozzle tips. ${ }^{6}$ All PODs in our study had rounded nozzle tips.

One of the limitations of the present study was user bias, as the samples collected for the study were labeled from the beginning. As a result, users were likely to be more cautious in using the ophthalmic drops, which underestimated the true prevalence of microbial contamination in PODs. Another limitation was that mobilization of the labeled PODs may have occurred in the OOC, resulting in missing bottles on the day of collection. Our study showed that only $3 \%$ of the risk could be predicted by the type of PODs and longer duration of use. Therefore, we would like to suggest further studies to address the various factors that could contribute to POD contamination, such as handling technique, the angle of instillation and bottle geometry. Other factors such as the temperature and humidity at which the PODs were stored, the frequency of use of the PODs in each patient and the number of patients per POD bottle may also be investigated. The physical appearance of the PODs tips and inner sides of the caps on the day of collection can also provide additional information to determine whether or not contamination is present.

Overall, the present results are consistent with previously reported findings showing that prolonged use of PODs by multiple users alone does not affect contamination rates. $5,10,17$

\section{Conclusion}

The dropper tip was more contaminated than the residual bottle contents, and the main contaminant was coagulasenegative Staphylococcus species, which are common commensal flora of the ocular conjunctiva and skin.

\section{Acknowledgments}

We would like to thank the staff at the ophthalmology outpatient clinic of UKMMC for providing us with the samples from newly opened and used bottles of the eyedrops. The assistance of the microbiology assistant at the lab is highly appreciated. We are grateful to Dr. Wan Rozita for her help with data analysis using SPSS and to
Prof. Dr. Syed Zulkifli for his help with the sample size calculation.

\section{Funding}

This study was funded by student grant under postgraduate studies from Universiti Kebangsaan Malaysia. The publication fee was funded by research grant from Universiti Malaysia Sabah.

\section{Disclosure}

This manuscript has not been presented elsewhere. No competing interests exist for any authors.

\section{References}

1. Jensen MK, Nahoopii R, Johnson B. Using multidose eyedrops in a health care setting: a policy and procedural approach to safe and effective treatment of patients. JAMA Ophthalmol. 2013;132:1476-1479. doi:10.1001/jamaophthalmol.2014.3248

2. Somner JE, Cavanagh DJ, Wong KK, Whitelaw M, Thomson T, Mansfield D. The precautionary principle: what is the risk of reusing disposable drops in routine ophthalmology consultations and what are the costs of reducing this risk to zero? Eye. 2010;24:361-363. doi:10.1038/eye.2009.129

3. Rahman MQ, Tejwani D, Wilson JA, Butcher I, Ramaesh K. Microbial contamination of preservative free eye drops in multiple application containers. $B r \quad J$ Ophthalmol. 2006;90:139-141. doi:10.1136/bjo.2005.078386

4. Tsegaw A, Tsegaw A, Tefera Abula YA. Bacterial contamination of multi-dose eye drops at ophthalmology department, University of Gondar, northwest Ethiopia. Middle East Afr J Ophthalmol. 2017;24:81-86. doi:10.4103/meajo.MEAJO_308_16

5. Livingstone DJ, Hanlon GW, Dyke S. Evaluation of an extended period of use for preserved eye drops in hospital practice. $\mathrm{Br}$ J Ophthalmol. 1998;82:473-475. doi:10.1136/bjo.82.5.473

6. Fazeli MR, Beheshtnezhad H, Mehregan H, Elahian L. Microbial contamination of preserved ophthalmic drops in outpatient departments: possibility of an extended period of use. DARU J Pharm Sci. 2004;12:151-155.

7. Danny H, Jokl K, Wormser GP, Nicholas NS, Montecalvo MA, Karmen CL. Bacterial contamination of ophthalmic solutions used in an extended care facility. Br J Ophthalmol. 2007;91:1308-1310. doi:10.1136/bjo.2007.115618

8. Sangeetha J, Prasanth DA. Bacterial contamination of in-use ocular medications. IOSR J Pharm Biol Sci. 2017;12:96-100.

9. Nentwich MM, Kollmann KH, Meshack J, Ilako DR, Schaller UC. Microbial contamination of multi-use ophthalmic solutions in Kenya. Br j Ophthalmol. 2007;91:1265-1268.

10. Feghhi M, Zarei Mahmoudabadi A, Mehdinejad M. Evaluation of fungal and bacterial contaminations of patient-used ocular drops. Med Mycol. 2008;46:17-21. doi:10.1080/13693780701487979

11. da Costa AX, Yu MCZ, de Freitas D, et al. Microbial cross-contamination in multidose eyedrops: the impact of instillation angle and bottle geometry. Transl Vis Sci Technol. 2020;9:7. doi:10.1167/tvst.9.7.7

12. Kim MS, Choi CY, Kim JM, Chung HR, Woo HY. Microbial contamination of multiply used preservative-free artificial tears packed in reclosable containers. $\mathrm{Br} j$ Ophthalmol. 2008;92:1518-1521. doi:10.1136/bjo.2008.144469 
13. Clark PJ, Ong B, Stanley CB. Contamination of diagnostic ophthalmic solutions in primary eye care settings. Mil Med. 1997;162:501-506. doi:10.1093/milmed/162.7.501

14. Tatham AJ, Sarodia U, Gatrad F, Awan A. Eye drop instillation technique in patients with glaucoma. Eye. 2013;27:1293-1928. doi:10.1038/eye.2013.187

15. Vandepitte J, Verhaegen J, Engbaek K, et al. Basic Laboratory Procedures in Clinical Bacteriology. World Health Organization. 2003.
16. Schein OD, Hibberd PL, Starck T, Baker AS, Kenyon KR. Microbial contamination of in-use ocular medications. Arch Ophthalmol. 1992;110:82-85. doi:10.1001/archopht.1992.01080130084030

17. HØvding G, Sjursen H. Bacterial contamination of drops and dropper tips of in-use multidose eye drop bottles. Acta Ophthalmol. 1982;60:213-222. doi:10.1111/j.1755-3768.1982.tb08375.x

\section{Publish your work in this journal}

Clinical Ophthalmology is an international, peer-reviewed journal covering all subspecialties within ophthalmology. Key topics include: Optometry; Visual science; Pharmacology and drug therapy in eye diseases; Basic Sciences; Primary and Secondary eye care; Patient Safety and Quality of Care Improvements. This journal is indexed on PubMed

Submit your manuscript here: https://www.dovepress.com/clinical-ophthalmology-journal
Central and CAS, and is the official journal of The Society of Clinical Ophthalmology (SCO). The manuscript management system is completely online and includes a very quick and fair peer-review system, which is all easy to use. Visit http://www.dovepress.com/ testimonials.php to read real quotes from published authors. 